\title{
Standard Practice for Dosimetry of Proton Beams for use in Radiation Effects Testing of Electronics
}

\author{
Margaret A. McMahan, Member, IEEE, Ewart Blackmore, Member, IEEE, Ethan W. Cascio, \\ Member, IEEE, Carlos Castaneda, Barbara von Przewoski, and Harvey Eisen, Senior Member, IEEE
}

\begin{abstract}
Representatives of facilities that routinely deliver protons for radiation effect testing are collaborating to establish a set of standard best practices for proton dosimetry. These best practices will be submitted to the ASTM International for adoption.
\end{abstract}

\section{INTRODUCTION}

Protons are frequently the dominant source of radiation in the space environment causing radiation effects such as single event effects (SEE), total ionizing dose effects (TID) or displacement damage (DD) to electronics or materials. Proton beams from particle accelerators are routinely used for accelerated ground testing of such radiation effects on devices to predict their performance in space. Standards exist for proton dosimetry for medical therapy facilities [1], but no equivalent standards have been established for dosimetry when protons are used for radiation effects testing. In the latter case, more diversity of energy, beam intensity and other operating conditions are required, which can lead to challenges in validation of the test conditions and dosimetry.

Under the auspices of the ASTM International Committees on Electronics (F11) and Dosimetry (E10) and the Space Parts Working Group's Hardness Assurance Committee, representatives of several North American facilities that routinely deliver protons for radiation effect testing of electronics are collaborating to establish a set of standard best practices for proton dosimetry. These best practices will be submitted to ASTM for adoption. The procedures to be considered include methods for measurement of fluence or dose, flux density, uniformity and energy of the proton beam,

Manuscript received July 3, 2008.

M.A. McMahan (corresponding author) is with the Lawrence Berkeley National Laboratory, Berkeley, CA 94720 USA (telephone: 510-486-5980, e-mail: p_mcmahan@lbl.gov).

E.W. Blackmore is with TRIUMF, Vancouver, BC V6T2A3 Canada email: blackmore@ triumf.ca).

E.W. Cascio is with Massachusetts General Hospital Cambridge, MA USA(e-mail: ecascio@partners.org).

C. Castaneda is with University of California Davis, Davis, CA USA (email(Castaneda@crocker.ucdavis.edu)

B. von Przewoski is with Indiana University Cyclotron Facility, Bloomington, IN USA (e-mail: bvonprze@indiana.edu)

H. Eisen is an independent consultant and Chair of the Space Parts Working Group Hardness Assurance Committee (e-mail: harveyeisen@aol.com) as well as potential interferences, e.g. slit scattering and/or secondary neutron production.

Testing using proton energies ranging from 10 to 1000 $\mathrm{MeV}$ can be done in air, and is typically used for radiation testing of electronics. Proton testing at energies below 10 $\mathrm{MeV}$ is becoming increasingly important to the space testing community and will be addressed in the standard practice; however, the fact that it must be carried out in vacuum adds complications and will not be addressed in this paper.

The beam intensities for simulating space and terrestrial effects range from approximately $10^{2}$ to $10^{11}$ protons $\mathrm{cm}^{-2} \mathrm{~s}^{-1}$. This large range is due to the very diverse needs of the community for proton testing of electronics - from low dose SEE studies in microelectronics to TID measurements in optical components. Many facilities limit the beam intensity because of inadequacies in the facility shielding, or in order to minimize activation of equipment and beamline components.

For electronics testing purposes the proton fluence or dose should be determined to better than $5 \%$. The proton beams should be monoenergetic or near monoenergetic $(\Delta \mathrm{E} / \mathrm{E}<5 \%)$ and the energy should be known to $<2-5 \%$ depending on the application. The beam flux density should be uniform to within 5-10\% across the size of the device under test (DUT).

There are a number of acceptable methods and techniques for determination of the fluence or dose, energy, energy spread and uniformity of the proton beam. These depend on type of accelerator, proton energy and beam size, and other experimental considerations. Test facilities require a combination of these methods for a full characterization of the proton beam.

\section{ELEMENTS OF STANDARD PROTON DOSIMETRY}

\section{A. Determination of dose or fluence}

Depending on the application, it may be most appropriate or even required to measure the effect of radiation as a function of either dose or fluence. This can be especially true if the device performance is either particularly dose dependent (e.g. TID effects) or particularly flux dependent (e.g. enhanced low dose rate sensitivity (ELDRS) effects). 


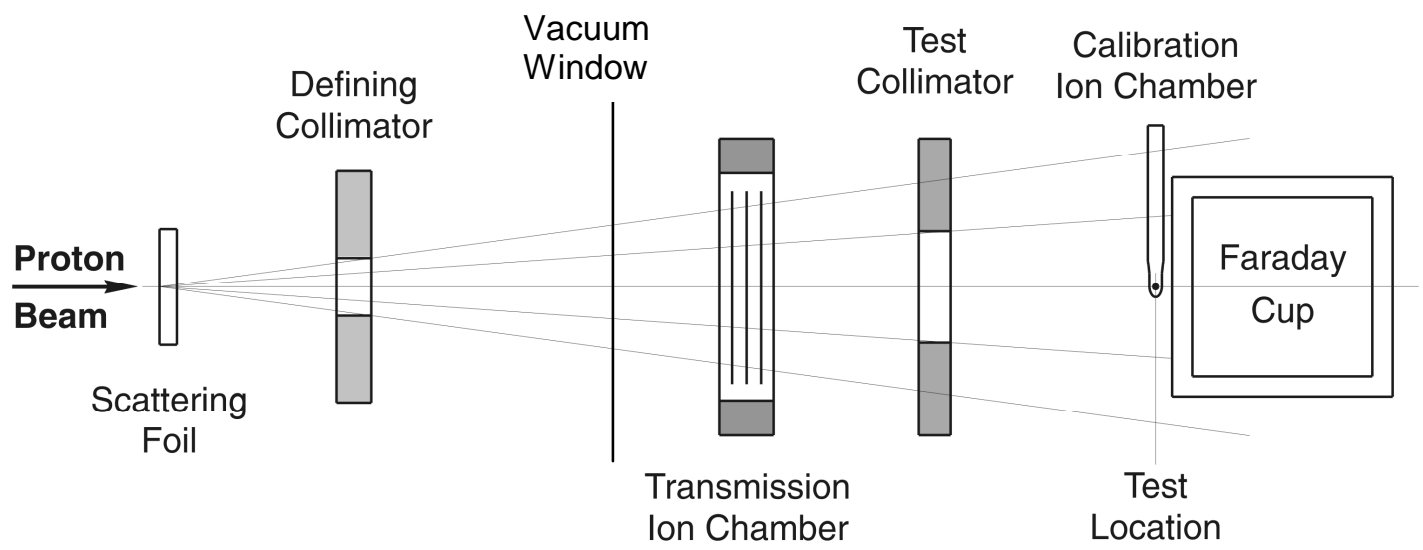

Fig 1. Typical experimental set up for proton test and dosimetry

\section{1) Relation between dose and fluence}

The relationship between dose and fluence is given by the equation:

$$
\text { Dose }(\text { rads })=\text { Fluence } * \text { LET } * 1.60 \times 10^{-8}
$$

where the fluence is in units of protons $/ \mathrm{cm}^{2}$ and the LET in $\mathrm{MeV} / \mathrm{g} / \mathrm{cm}^{2}$.

\section{2) Monitoring dose or fluence}

Various kinds of detectors can be used to either directly monitor the dose or fluence, to calibrate the monitor detector or to determine other beam characteristics such as uniformity, energy and energy width. The dose or fluence is monitored with some kind of transmission detector that is larger than the maximum collimator size and DUT and thin enough to minimize scattering and energy degradation. Depending on proton energy and the beamline configuration, the typical transmission dosimeter is either a transmission ion chamber [TIC] (usually operated in air) or a secondary emission monitor [SEM] (operated in vacuum). A typical set up for in-air testing using a TIC is shown in Figure 1. The only difference in the case of dosimetry using a SEM would be the location of the vacuum window. Transmission detectors can be configured to give beam uniformity information as well as dose.

Plane-parallel, or transmission, ion chambers provide an accurate and reproducible method of measuring the proton dose rate or total dose and from this measurement determining the proton flux or fluence. An introduction to ion chamber technology can be found in Knoll [2]. An ion chamber consists either of a foil at high voltage centered between two thin signal foils at ground, or the opposite configuration, with a center signal foil stretched between two high voltage foils. The intervening gaps are filled with air or an inert gas at some known pressure, often atmospheric. Particles traversing the foils ionize the gas, and either the electrons or ions are collected at the ground, or collector, foil. Ion chambers are characterized by the quantity $\mathrm{W}$, defined as the amount of energy it takes to create an ion pair in the gas. $\mathrm{W}$ depends on the particle detected and the choice of gas; for protons, it is 30-35 $\mathrm{eV} /$ ion pair for all commonly used gases. Ambient air ion chambers are the simplest but may require a correction on the order of a few percent for changes in pressure and temperature.

TICs are typically operated at an applied voltage high enough so that recombination of the ion pairs created in the gas are negligible; this is determined using beam. The voltage is increased until the current versus voltage curve reaches a plateau region. Under these conditions the current is directly proportional to the proton flux. The high voltage may be of either polarity depending on the chamber configuration and will vary from $100 \mathrm{~V}$ to 2000 $\mathrm{V}$, depending on the distance between foils and proton peak intensity.

The small ion chamber current generated is typically read out through a recyling integrator module. This outputs a digital pulse for every $10^{-6}-10^{-11}$ Coulombs of input charge. The integrator output is then read into a scaler. The scaler readout is proportional to the beam flux times the total energy lost in the gas (approximated by LET multiplied by the thickness, $\mathrm{t}$ ):

$$
\text { Counts } \sim \mathrm{I}_{\mathrm{p}} * \mathrm{LET}_{\text {gas }} * \mathrm{t} / \mathrm{W}
$$

In converting to dose at the DUT, a correction must be made for the difference in LET for the proton traversing the gas compared to the composition of the DUT.

Ion chambers can be made position sensitive by dividing one or more of the foils. For example, some ion chambers made in the two signal foil configuration are divided into a series of concentric rings on one side for tuning beam diameter and uniformity, and quadrants on the second foil for beam centering.

TICs are limited at low fluxes by the sensitivity of the recycling integrator, and at high fluxes by saturation in the gas caused by recombination of the ion pairs. Typical operating ranges are given in Table 1.

A multi-foil secondary electron emission monitor (SEM) can also be used to monitor the proton beam current. An odd number $(\geq 3)$ of copper or aluminum foils are stacked and biased such that odd foils are at positive bias while even foils are connected to an electrometer. The voltage 
needed increases with the foil spacing. The secondary electron current depends on the energy of the protons and the number of foils used. The higher the energy of the proton beam the fewer electrons will be produced. This effect can be compensated for by the use of more foils. A SEM is suitable for monitoring proton beam currents as low as 10 picoamperes $\left(6.2 \times 10^{7}\right.$ protons/s $)$. A beam defining collimator placed just upstream of the SEM is required in order to precisely define the diameter of the beam.

Facilities that use TICs as the primary dosimetric tool must rely on other devices for measuring dose or fluence at low fluxes. This is most commonly a scintillating material of some kind coupled to a photomultiplier tube. [3] Organic scintillators such as fast plastics - when configured correctly and operated in a light-free environment - can be sensitive to very low ion fluxes up to approximately $10^{6}$ protons/s.

\section{3) Calibration of dosimeters}

The preferred means of calibrating a dosimeter depends on whether the measurement is being performed in fluence or dose. For fluence, a calibration should be a measurement of direct charge or current. For this the most straightforward device is a Faraday cup. For dose measurements, the calibration is typically accomplished using a standard reference ion chamber that has been calibrated externally. In either case, it is preferable for the calibration to be performed at the location of the DUT. If the calibration device is located elsewhere, e.g., a Faraday cup in the beamline, great care must be taken to ensure that the flux density does not change between the calibration device and the monitoring device.

A Faraday cup (FC) is an excellent method of confirming the fluence calibration by direct measurement. Because it completely stops the proton beam it cannot be used during testing. Useful references on Faraday cup design are given in Refs. [4-5]. The FC requires a graphite or heavier metal beam absorber that is insulated from the ground container. From a radiation safety perspective, graphite has the advantage of reducing radioactivation but the disadvantage that for high intensity beams, it has been known to sputter, resulting in loose activity, or contamination. In addition, it may not be practical above proton energies of $200 \mathrm{MeV}$ because of its low density. A guard ring or equivalent structure is required to reduce current leakage from the high voltage and a suppressor ring or magnetic field is required to remove or collect secondary electrons that come from proton interactions in the entrance foil or absorber.

The FC system should operate in a high vacuum, either within the proton beam line or as a separate device with a thin beam entrance window and its own vacuum system. (The latter is necessary if the FC is to be used at the position of the DUT.) Alternatively, simple non-vacuum Faraday cups can also be constructed by surrounding a sufficiently thick target with an insulator (such as Kapton) and a shield. These have been shown to agree with standard (vacuum) Faraday cups to $\sim 1-2 \%$.

The calibration ion chamber is used to measure the dose at the device test location. The active volume of the chamber should be smaller than the beam size. Examples from medical applications [6] are the Exradin or Farmer series of thimble chambers. A typical calibration for a small chamber is $60 \mathrm{rads} / \mathrm{nC}$ and transverse dimensions are about $0.125 "$ ( $3 \mathrm{~mm}$ ). This chamber is filled with ambient air so a correction for temperature and pressure is needed for accurate work. A standard ${ }^{60} \mathrm{Co}$ calibration factor can be provided by the supplier and converted to a proton calibration using a prescribed technique. The calibration of the reference chamber can also be independently verified against a Faraday cup using Equation 1.

\section{B. Achieving and determining Beam Uniformity}

Proton beams for radiation testing are often delivered over a large physical area, either to enable testing of multiple components at the same time, or to ensure that the central portion of the beam is uniform, or both. This requires that the dose monitoring device be as large or larger in area than the part of the beam which is to be utilized. Standard focusing magnets employed in most accelerator facilities result in a beam that has a Gaussian distribution in space, but which maintains any nonuniformities it had upon exiting the accelerator. The beam may be made more uniform through the use of scattering foils then defocused with quadrupole magnets. In general, the beam size should be large so that only the central $10 \%$ of the beam is used, in order to obtain maximum uniformity. The best uniformity or transverse distribution one can achieve with these techniques is on the order of 5$10 \%$.

In order to achieve better efficiency, thus allowing more of the beam to be directed to the device (important for high dose experiments), other techniques have been developed which are generally more expensive and require more set up time. These include the use of a second, contoured scattering foil in order to scatter beam from the center region into the outer portion, thus filling in the shoulders of the beam distribution, leading to a "flattop" beam profile. Beam rastering - in which a focused beam is moved in both the vertical and horizontal axes across the sample can also be employed.

It is possible to make the primary dosimetric device, whether TIC or SEM, position-sensitive. This enables adjusting uniformity during beam tuning. Alternatively, an array of small detectors such as silicon diodes can be employed to check uniformity after tuning, or one such detector can be moved across the beam in the $\mathrm{x}$ - and $\mathrm{y}-$ dimensions.

The beam uniformity can also be measured using radiosensitive film; Gafchromic ${ }^{\mathrm{TM}}$ film is used by most facilities. The film is exposed to the proton beam for a dose of 2-100 krad (20-1000 Gy) depending on the 
sensitivity of the film and the proton energy. The film can be read with a densitometer or scanned using an optical scanner. Gafchromic film has been explored as a possible dose monitor as well, but has been shown to be too sensitive to proton energy to use as a quantitative device.[7]

\section{Energy and Energy Straggling}

The uncertainty in the energy of an accelerated particle depends on the type of accelerator but is generally known to better than $1 \%$, even better for tandem accelerators. This uncertainty is negligible compared to the uncertainty in the energy at the DUT after the proton has traversed the scattering foil, the exit window and air gaps in addition to the monitoring device (TIM or SEM) and any degraders. The energy loss in these materials can be calculated using modern stopping power codes such as SRIM 2006 [8] SRIM gives average uncertainties in LET value - when calculations are compared to experimental data - of $8.5 \%$ for protons on $\mathrm{Si}$, for example, at energies less than 10 $\mathrm{MeV}$, and of approximately $4 \%$ above $10 \mathrm{MeV}$. [8] For thick absorbing material such as degraders, the energy loss must be integrated over the thickness of the material.

Alternatively there are various standard techniques to either directly or indirectly measure the energy at the DUT position. At low energies, silicon detectors or diodes are employed. Silicon has a very linear energy response for protons and good energy resolution, but is limited to lower energies by available thicknesses. Scintillators can also be used to directly measure the energy. Scintillators are available thick enough to stop most proton energies; however, the energy response is not linear. The energy response of protons has been well studied for several common organic and inorganic scintillators. [3] More sophisticated techniques - such as use of a multi-leaf Faraday cup - may be employed to measure the energy straggling after passing through thick absorbers such as energy degraders.

1) Energy straggling

If the particle traverses enough material to significantly change the energy, e.g. energy degraders, then the beam energy will spread. This is called energy straggling. The amount of straggling can be calculated using SRIM 2006 [8] or measured directly.

\section{2) Energy straggling in a degrader}

Degraders are often used to reduce the energy of the beam, enabling faster and more frequent energy changes. The energy spread of the degraded beam depends on range straggling in the degrader (an irreducible effect) and on the initial energy spread (an accelerator and beamline dependent effect) according to

$$
\sigma^{2}=\sigma_{S T R}^{2}+\left(\frac{S_{2}}{S_{1}} \sigma_{I N T}\right)^{2}
$$

where $\sigma_{\mathrm{INT}}$ is the initial energy spread of the proton beam before the degrader, $\sigma_{\mathrm{STR}}$ is the range straggling due to multiple path lengths in the degrader itself, $S_{1}$ is the stopping power of the degrading material for protons at the initial energy, and $S_{2}$ is the stopping power of the degrading material for protons at the final energy. Analyzing magnets located between the degrader and the endstation can serve to narrow the energy width of the beam delivered to the DUT, at the expense of beam intensity.

\section{3) Measuring the energy straggling}

If the resolution of the detector is high enough, any detector that directly measures the beam energy, e.g. a $\mathrm{Si}$ or scintillator detector, will also determine its spread. The detector is placed in the beam at the location of the device under test (DUT), in order to determine the beam spread due to all sources, including air. This method is limited to low fluxes; see Table 1 for the maximum flux density for the various kinds of energy detectors.

The measured width of the peak has two contributions that must be considered. The actual energy spread $\sigma$ as defined in (3) and the instrumental resolution of the energy detector itself $\sigma_{\mathrm{RES}}$. The total measured peak width $\sigma_{\mathrm{MEA}}$ is given by

$$
\sigma_{M E A}^{2}=\sigma^{2}+\sigma_{R E S}^{2}
$$

A standard method of estimating the contribution from $\sigma_{\text {RES }}$ is to measure the width of the peak produced by the $0.662 \mathrm{MeV}{ }^{137} \mathrm{Cs}$ source, typically a few a few tens of $\mathrm{keV}$. The instrumental resolution is then assumed to vary with energy as $\mathrm{E}^{-1 / 2}$, about the value found at $0.662 \mathrm{MeV}$. The resulting corrections are typically on the order of a few percent.

At fluxes above $\sim 10^{5}$ protons $/ \mathrm{cm}^{2} / \mathrm{s}$ a multi-leaf Faraday Cup (MLFC) may be used. A MLFC consists of a number of conducting ( $\mathrm{Al}$ or $\mathrm{Cu}$ ) sheets or leaves separated by insulating layers. For example 30 layers of $0.5 \mathrm{~mm}$ thick $\mathrm{Al}$ leaves alternating with $80 \mu \mathrm{m}$ thick kapton sheets sandwiched between a front and a back cover plate make a useful MLFC. As the current is read out from each conducting layer, the highest current is measured where the protons stop. The beam energy can then be determined from the number of layers that the protons are able to penetrate.

The MLFC has to be thick enough that the protons stop within its sensitive volume. For higher proton energies additional copper degraders are placed in front of the MLFC. An MLFC can resolve energy spreads of $\sim 1 \mathrm{MeV}$.

The beam energy is then determined from an energy loss calculation where the incident energy at the location of the DUT is treated as a parameter that is optimized such that the measured MLFC spectrum is reproduced. The energy loss calculation introduces an uncertainty of up to a few percent due to the specific iteration method used. Uncertainties in the calculations increase with energy lost or material thickness. 
The asymmetry of the peak becomes more pronounced at lower beam energies and is due to the non-linearity of the stopping power as a function of energy. The tail becomes more pronounced at higher beam energies, due to secondary effects such as are discussed in the next section.

\section{INTERFERENCES}

\section{A. Collimation/slit scattering}

Scattering of the proton beam from the edges of collimators and slits will lead to errors in the dosimetry, particularly if there is a collimator between the dosimeter and the DUT. This effect will be larger for higher energy beams that require thicker collimators or slits. It has been observed for the facility at TRIUMF to be on the order of a few percent. [9]

Slit scattering has been well studied for many years both theoretically and experimentally $[10,11]$ and can be minimized by careful design of the collimators or slits.

\section{B. Energy Loss and scattering within the DUT}

Another source of error in the dosimetry will arise from self-scattering and energy loss within the DUT itself. This must be considered particularly in devices that are very thick or have very thick overlayers.

\section{Secondary Neutron Contamination}

Secondary neutrons are an inevitable byproduct of the proton beam interacting with matter. Thus there will always be some secondary neutron fluence in a proton beamline and the local test area. Typical sources of secondary neutrons are the collimating apertures used to define the beam spot, energy degraders used to reduce the proton energy, and the final beam stop. Secondary neutrons can also be produced in the device under test itself. While the flux of secondary neutrons is typically much lower than the proton flux delivered to the test target, these secondary neutrons can still affect the test process in a number of ways. They can cause SEE (Single Event Effects) in the device under test or in other components located on the same board as the test device. They can also cause upsets in ancillary support equipment located close to the test area, such as computers used to monitor the test. Secondary neutron production was measured for $230 \mathrm{MeV}$ protons at the Northeast Proton Therapy Center [12] and has been measured at other facilities as well.

Neutrons in two energy ranges are of particular concern when testing electronics. Neutrons with energies above 20 $\mathrm{MeV}$ have a similar SEE cross-section as protons of the same energy [13]. Very low energy, or thermal, neutrons, those with energies ranging from $0.025 \mathrm{eV}$ to around 10 $\mathrm{eV}$, can react with ${ }^{10} \mathrm{~B}$, an isotope of boron used in the fabrication of some chips. This reaction produces products with sufficient energy to cause SEE effects in these chips [14]. On the other hand, neutrons in the $\mathrm{keV}$ range have very little effect on electronics. It is thus useful to have quantitative information about both the fluence and the energy spectrum of the secondary neutrons produced when considering any possible effects on the test procedure and results.

The number of secondary neutrons can be kept to a minimum in the design of the beamline and irradiation facility by best practices such as locating energy degraders upstream of the final bending magnet and locating the beam stop far from the position of the DUT. Thermal neutrons can be shielded using cadmium.

\section{CONCLUSIONS}

We have identified a number of best practices for determination of the fluence or dose, energy, energy spread and uniformity of a proton beam to be used in radiation effects testing. On the other hand, there are many acceptable detectors and techniques that can be used to accomplish accurate and reliable dosimetry and characterization of the proton beam. The test facilities collaborating to establish standard best practices through ASTM International are listed in Table 2.

\section{V.REFERENCES}

[1] "Clinical proton dosimetry Part 1: Beam production, beam delivery and measurement of absorbed dose", International Commission on Radiation and Units, Report 59 Bethesda, MD,

[2] G. F. Knoll, "Radiation Detection and Measurement", John Wiley and Sons, New York, 1979, pp. 155-173

[3] J.B. Birks, "The Theory and Practice of Scintillation Counting", Pergamon Press, Oxford (1964).

[4] J.F. Zeigler, et al., "Portable Faraday Cup for nonvacuum proton beams", IBM Journal of Research and Development, Vol. 40, January 1996.

[5] R. Cambria, et al., "Proton beam dosimetry: A comparison between the Faraday cup and an ionization chamber", Phys. Med. Biol. 42, 1185 (1997).

[6] "Absorbed Dose Determination in External Beam Radiotherapy, An International Code of Practice for Dosimetry based on Standards of Absorbed Dose to Water", International Atomic Energy Agency, IAEA TRS-398, 2001.

[7] A.E. Buenfill et al., "Response of radiochromic dye films to low energy heavy charged particles", Nucl Instr Meth B197, 317 (2002).

[8] SRIM 2006: Stopping and Range of Ions in Matter www.srim.org

[9] E.W. Blackmore, private communication

[10]F.G. Resmini et al., "Slit scattering effects with medium energy alpha particles and protons", Nucl Instr Meth 74, 261 (1969).

[11] J. Nickel, "Slit scattering in a microbeam set-up", Nucl Instr Meth B225, 402 (2004).

[12] E.W. Cascio et al., "Secondary neutron fluence in radiation test beams at The Northeast Proton Therapy Center", Proceedings of the 2005 IEEE Radiation Effects Data Workshop, Seattle, WA, 2005, pp. 175178. 
[13] P.E. Dodd et. al., proceedings of the 2002 Int. Elect. Device Meeting, 2002, pp. 333-336.

[14] R.C. Baumann and E.B. Smith, "Neutron-induced Boron Fission as a Major Source of Soft Errors in Deep Submicron SRAM Devices", IEEE Proc. IRPS, 2000, p. 297.

[15]M.B. Johnson et al., "Berkeley Accelerator Space Effects (BASE) Light Ion Facility Upgrade", Proceedings of the 2006 IEEE Radiation Effects Data Workshop, Ponte Verdra Beach, FL, 2006, pp. 183187

[16] K.M. Murray et al., "Calibrated charged particle radiation system with precision dosimetric mearurement and control", Nucl Inst Meth A281, 1989, pp. 616-621.

[17] C.M. Castaneda, "Crocker National Laboratory (CNL) Radiation Effects Measurement and Test Facility", Proceedings of the 2001 IEEE Radiation Effects Data Workshop, Vancouver, BC, Canada, 2001, pp.77-81
[18] E.W. Blackmore, "Operation of the TRIUMF (20-500 $\mathrm{MeV}$ ) proton irradiation facility", Proceedings of the 2000 IEEE Data Workshop, 2000, pp. 1-5.

[19]E.W. Blackmore, "Improved capabilities for proton and neutron irradiations at TRIUMF", Proceedings of the 2003 IEEE Radiation Effects Data Workshop, Monterey, CA, 2003, pp. 149-155.

[20]B. von Przewoski et al., "Beam properties of the new Radiation Effects Research Stations at Indiana University Cyclotron Facility", Proceedings of the 2004 IEEE Radiation Effects Data Workshop, Atlanta, GA, 2004, pp. 145-150.

[21]E.W. Cascio et al., "The Proton Irradiation Program at the Northeast Proton Therapy Center", Proceedings of the 2003 IEEE Radiation Effects Data Workshop, Monterey, CA, 2003, pp. 141-144. 
TABLE 1. SUMMARY OF DETECTORS DisCUSSED IN THIS STANDARD PRACTICE

\begin{tabular}{|c|c|c|}
\hline \multirow[t]{2}{*}{ Type of detector } & \multicolumn{2}{|c|}{ Flux or dose range } \\
\hline & $\mathrm{E}=10-65 \mathrm{MeV}$ & $\mathrm{E}=65-500 \mathrm{MeV}$ \\
\hline \multicolumn{3}{|c|}{ Dose or Fluence monitors: } \\
\hline $\begin{array}{l}\text { Transmission Ion } \\
\text { Chamber (TIC) }\end{array}$ & $10^{6}-10^{10} \mathrm{p} / \mathrm{cm}^{2} / \mathrm{s}$ & $10^{6}-10^{10} \mathrm{p} / \mathrm{cm}^{2} / \mathrm{s}$ \\
\hline $\begin{array}{l}\text { Secondary Electron } \\
\text { Monitor (SEM) }\end{array}$ & $10^{7}-10^{11} \mathrm{p} / \mathrm{cm}^{2} / \mathrm{s}$ & $10^{7}-10^{11} \mathrm{p} / \mathrm{cm}^{2} / \mathrm{s}$ \\
\hline Scintillator (SC) & $10^{3}-10^{6} \mathrm{p} / \mathrm{cm}^{2} / \mathrm{s}$ & $10^{3}-10^{6} \mathrm{p} / \mathrm{cm}^{2} / \mathrm{s}$ \\
\hline \multicolumn{3}{|c|}{ Calibration of dose or fluence: } \\
\hline $\begin{array}{l}\text { Thimble Ion Chamber } \\
\text { (IC) }\end{array}$ & $1-500 \mathrm{~Gy}$ & $1-500 \mathrm{~Gy}$ \\
\hline Faraday Cup (FC) & $10^{7}-10^{12} \mathrm{p} / \mathrm{cm}^{2} / \mathrm{s}$ & $10^{7}-10^{10} \mathrm{p} / \mathrm{cm}^{2} / \mathrm{s}$ \\
\hline \multicolumn{3}{|c|}{$\begin{array}{l}\text { Energy or energy straggling: } \\
\qquad 10^{2}-10^{4} \mathrm{p} / \mathrm{cm}^{2} / \mathrm{s}\end{array}$} \\
\hline $\begin{array}{l}\text { Multi-leaf Faraday cup } \\
\text { (MLFC) }\end{array}$ & $10^{6}-10^{12} \mathrm{p} / \mathrm{cm}^{2} / \mathrm{s}$ & $10^{6}-10^{12} \mathrm{p} / \mathrm{cm}^{2} / \mathrm{s}$ \\
\hline Segmented TIC & $\begin{array}{l}\text { Uniformity: } \\
10^{6}-10^{10} \mathrm{p} / \mathrm{cm}^{2} / \mathrm{s}\end{array}$ & $10^{6}-10^{10} \mathrm{p} / \mathrm{cm}^{2} / \mathrm{s}$ \\
\hline Gafchromic film (GF) & $1-1000 \mathrm{~Gy}$ & $1-1000 \mathrm{~Gy}$ \\
\hline
\end{tabular}

TABLE 2.

SUMMARY OF PROTON IRRADIATION FACILITIES PARTICIPATING IN THIS EFFORT

\begin{tabular}{|c|c|c|c|c|c|c|}
\hline Facility & $\begin{array}{c}\text { Primary } \\
\text { beam } \\
\text { energy } \\
\text { /range } \\
(\mathrm{MeV})\end{array}$ & $\begin{array}{l}\text { Degraded beam } \\
\text { energy/ range } \\
(\mathrm{MeV})\end{array}$ & $\begin{array}{l}\text { Available fluxes } \\
\text { (protons } / \mathrm{cm}^{2} / \mathrm{s} \text { ) }\end{array}$ & $\begin{array}{c}\text { Beam } \\
\text { diameter }(\mathrm{cm})\end{array}$ & $\begin{array}{l}\text { Primary Dosimetry/Beam } \\
\text { Characterization Tools }\end{array}$ & $\begin{array}{c}\text { Secondary Calibration } \\
\text { Tools }\end{array}$ \\
\hline $\begin{array}{c}\text { 88-Inch Cyclotron, } \\
\text { Berkeley, CA, USA } \\
{[15]} \\
\end{array}$ & $12-55$ & N.A. & $10^{2}-10^{10}$ & $2-15$ & $\begin{array}{l}\text { TIC (dose, uniformity), GF } \\
\text { (uniformity), SD (energy), SC } \\
\text { (fluence) }\end{array}$ & FC, IC \\
\hline $\begin{array}{c}\text { Crocker Laboratory, } \\
\text { Davis, CA, USA } \\
{[16,17]}\end{array}$ & $1-68$ & $1-68$ & $10^{2}-10^{11}$ & 6 & $\begin{array}{l}\text { SEM (dose, uniformity), GF } \\
\text { (uniformity) }\end{array}$ & $\mathrm{FC}$ \\
\hline $\begin{array}{c}\text { TRIUMF, } \\
\text { Vancouver, BC, } \\
\text { Canada }[18,19] \\
\end{array}$ & $65-500$ & $15-498$ & $10^{2}-10^{10}$ & $0.5-10$ & $\begin{array}{c}\text { SEM (dose), TIC (dose), FC } \\
\text { (fluence), GF (uniformity), } \\
\text { scanned SD (uniformity) }\end{array}$ & FC, IC \\
\hline $\begin{array}{c}\text { Indiana University } \\
\text { Cyclotron Facility, } \\
\text { Bloomington, IN, } \\
\text { USA [20] }\end{array}$ & 205 & $30-200$ & $10^{2}-10^{11}$ & $2-30$ & $\begin{array}{l}\text { SEM (dose), MLFC (energy, } \\
\text { energy spread), GF } \\
\text { (uniformity) }\end{array}$ & FC, IC \\
\hline $\begin{array}{l}\text { Northeast Proton } \\
\text { Therapy Center, } \\
\text { Cambridge, MA, } \\
\text { USA [21] }\end{array}$ & 230 & $20-230$ & $10^{2}-10^{1}$ & $1-30$ & TIC (dose) & FC, IC, SD \\
\hline
\end{tabular}

